EPJ Web of Conferences 58, 04001 (2013)

DOI: $10.1051 /$ epjconf/20135804001

(C) Owned by the authors, published by EDP Sciences, 2013

\title{
What kind of time for a Time Machine?
}

\author{
Marina Alfano ${ }^{1}$ and Rosolino Buccheri ${ }^{2}$ \\ ${ }^{1}$ Conservatorio Statale di Musica «Girolamo Frescobaldi», Largo Antonioni 1, 44121, Ferrara, Italy \\ ${ }^{2}$ ITD-CNR and IASF-INAF, Via Ugo La Malfa 153, 90146, Palermo, Italy
}

\begin{abstract}
The linear, unstructured, parameter $t$ used in the equations of mechanics, in spite of its great aptness in describing the nature's laws, does not fit with the unidirectional flow of time $t_{s}$ subjectively experienced by humans, just the investigators of nature. Being $t_{s}$ the main foundation upon which we build our knowledge of nature through our continuous and inescapable reciprocal interaction - the possible key factor of our cerebral modulation, mediator between us and the world - , its objective essence appears to be inevitably destined to remain unveiled. We derive that any imagined and theoretically possible Time Machine, aimed to get us in our past or in our future allowing us to act there, does not have any practical grounds if it is built by using the illusory, impersonal, time, modeled by the parameter $t$, at the place of our interpersonal lived time $t_{s}$. A real, humanly-tuned, Time Machine could perhaps arise by integrating $t_{s}$ in the body of a new kind of rationality - a 'complex thought' - where empiricism and logic-mathematic are harmonized with participation and interaction. Ongoing joint research in neurophysiology and physics (without neglecting any important contribution coming from anthropology) will surely help achieving such a goal.
\end{abstract}

\section{Evolution of our system of thought}

Our image of the world is based on the experience we have of it through our senses and through the subsequent elaboration by our brain of the gathered information. At the dawning of the civilization - the time of myth -, the acquisition and the elaboration of the experiential information were based on a process of co-oscillatory resonance established by man with his environment, as the effect of a hearing amplified by his body, transmitted to his emerging consciousness. An important feature of such participatory attitude of our ancestors was that of finding themselves totally immersed in the flow of time, seen, from within, as a sort of continuous present.

Along the evolution, the need to communicate generated the oral language, originally of a mimetic-poetic type (still pertaining to the effusive-participatory consciousness typical of the 'primitive mentality') and then of a dialectic type, whose «question-and-answer» structure shows already a separation between communicating individuals [1]. With writing - come after orality - the space -temporal bonds between the emitter and the receiver of the communication were reduced, thus extending the flair for abstraction and making less pregnant the possibility of an empathic resonance. As a result of such a flair for abstraction, an «extended consciousness» [2] eventually developed, culminating in the to-day's 'rational system of thought', mainly based on the categories of logics, mathematics and empiricism, typical of the science's approach to knowledge, and on a more precise consciousness about the flow of time, from past to future.

As a consequence of the acquired habit to the rational system of thought, many of those 'subjective' skills connected with the knowledge captured and processed in a participatory, undifferentiated, attitude, are nowadays forgotten or disregarded as 'irrational', despite the very fact that such a knowledge is constantly in support of our 'reason' for the solution of problems (sometimes vital ones) not attainable by using the usual logical tools.

\section{Limitations to our 'rationality'}

In this section we intend to show that our rational system of thought suffers several important shortcomings due to which it may be legitimate to be skeptic on the possibility to base only on it in order to really achieve a complete and 'objective' knowledge of the reality. We will see that, because of these shortcomings limiting the interpretation

This is an Open Access article distributed under the terms of the Creative Commons Attribution License 2.0, which permits unrestricted use, distribution, and reproduction in any medium, provided the original work is properly cited. 
of our experiences, it is not possible to raise to the rank of 'objective' truths all the conclusions of our rational system of thought.

In one of his essays, Michał Heller [3] identifies three aspects of our rationality:

- the rational structure of the Universe, accessible to science's mathematical-empirical methods;

- the 'reasoning', i.e. the use, in all our discussions, of logical tools apt to derive correct conclusions from clearly defined premises;

- the rational way of living, where the rules, although in principle in agreement with those of the 'reasoning', are very difficult to codify due to many deviating factors, including the aims we have in life and the means we use to achieve them.

As it can be clearly understood, the three aspects are not unrelated to each other, since our way of living is certainly able to affect somewhat our reasoning and, in a much lesser degree, also the way we investigate the Universe.

Furthermore, the degree of 'perfection' of the rational system of thought gradually decreases going from the first to the third aspect. The most important, unavoidable, reason for this lack of 'perfection' is due to the fact that the elaboration of the experience that we have of the world is biased by constraints of various kind, related both to our physical and psychical structure and to our relationship with the environment. We have identified two kind of constraints:

- 'soft' constraints, related mainly to single persons, showing great individual differences in beliefs, or related to social groups with differences in cultural traditions;

- 'hard' constraints, related to man's physical/psychical structure or to the structure of the system of thought itself, therefore intrinsic to man, independently of his belief or cultural tradition. In view of its fundamental role in the acquisition of knowledge, it seems to us that the hardest of all constraints may surely be the way in which the flow of time is lived by man, for its close connection with the degree of his separation from the environment.

\subsection{Bias by 'soft' constraints}

Soft constraints which filter our experiences may be identified among our cultural traditions, personal beliefs, wrong intuitions, pre-comprehensions and many other 'irrational' skills, built along the course of our life and gradually transformed into more or less rigid schemas of thought. We always use these schemas when we observe and interpret phenomena around us.

To this purpose, it is interesting to refer to Martin Heidegger when he states that an 'objective' knowledge is impossible because every knowledge is the result of an 'interpretation' on the basis of our pre-dispositions, previsions and pre-cognitions. He claims that the act of interpretation is never the result of a neutral knowledge of something 'objective', but only a representation of the interpreting subject; the external reality is never precisely measurable and may be unveiled by man only partially [4]. This concept was taken up by Hans George Gadamer who thought that the process of increasing knowledge is a sort of 'hermeneutic circle' where we have already, in advance, some knowledge of what we are going to learn. According to him, our comprehension cannot be reduced to a pure reproductive act as it has, instead, always a poietic, productive, component [5].

For what concerns the second aspect of the system of thought considered by Heller, many of the 'soft' constraints generally lose their effectiveness within our everyday experience, where empirical verifications are obtainable through the mediating interaction of different persons.

This mediation process, however, may take, in some cases, quite negative directions, leading to disastrous situations, stable for long time but with important faults and gaps in our knowledge. This may happen when an idea - incorrect but able, in particular social conditions, to charm large masses of people - is carried out and supported by charismatic and powerful personalities, as it has been clearly shown by several cases in the human history. Having this in mind, it is not unrealistic to hypothesize that the present world crisis might be due to important conceptual mistakes imposed by the strong opinion of 'experts' of economy or sociology.

The influence of 'soft' constraints is practically eliminated within the process of the nature's investigation by science (first aspect of the system of thought). In this case, due to the powerful collective approach used by science, any statement coming from within the scientific community, being the result of a critical check obtained by repeated observations and experiments, allows to escape the enormous quantity of individual differences in beliefs and cultural traditions among individual scientists. In the context of the scientific work, in fact, the good intuition of just one single scientist, carefully and repeatedly checked by the rest of the community, is enough to drive the process of knowledge, with very high speed, toward the correct direction. The outstanding achievements of the scientific method, capable to figure very precise models of representation of a huge region of the reality, directly observable and empirically verifiable/falsifiable, witnesses its general effectiveness.

\subsection{Bias by 'hard' constraints}

Among the 'hard' constraints - valid for the whole mankind and acting as a real selection effect everywhere in the Planet - we include the limitations implicit in the human physical and psychical structure together with the 
unavoidable restriction to perceive and 'rationally' analyze - through our habitual (conscious and unconscious) exchange of information with the surrounding world - almost only those phenomena occurring at our dimensional size. It has to be stressed here that this selection effect cannot be univocally defined for everybody of us, due to the great differences between individuals, both in the unconscious intuition skills and in the way the whole information is 'rationally' elaborated. At any rate, these constraints shape our 'common sense' and our language, drastically filtering our knowledge of the reality.

As a consequence, the concepts and the language, developed in accordance with a common sense shaped by all these filters, loses certainly their validity beyond the direct control of our sensory organs, where we cannot design selective and repeatable experiments aimed to empirical verifications.

However, it is essential to observe that, being man product and part of nature, the physical/psychical human structure potentially contains the possibility to unconsciously read the world in its deepest aspects. At this purpose, Gerald Schröder writes: "If the universe is indeed the expression of an idea, the brain may be the sole antenna with circuitry tuned to pick up the signal of that idea» [6, p. 105]. Our structure, in fact, may be subject, on the basis of man's actual needs, to 'unexpected' transformations capable to greatly widen his knowledge horizons. Such transformations may occur in conditions of a higher level of consciousness where our biases can be, almost partially, overcome.

The discovery of the limits of validity of our common sense within the domain of our daily experience, which fatally destroys our illusion about its universal effectiveness, is one of the greatest achievements of modern physics: beyond our dimensions, scientific experiments and observations cannot even be imagined and the result of the use of abstract mathematics, useful in many occasions, may otherwise reveals to be conjectural or even ineffectual. As far as we know from physics, this is generally the case of the knowledge about the microworld governed by quantum mechanics (not directly observable) and, at a much lesser extent, that of the knowledge about the macroworld (observable but only indirectly falsifiable), both described - when possible - by the abstract but powerful language of mathematics, the only way to make them accessible to us in some details. It is worth to specify, at this purpose, that some typical quantum phenomena may trespass the Planck threshold and manifest themselves at our dimensions (like, for example, wave-particle duality or non-locality of EPR events); in these cases, suitable experiments can be set up which, together with the use of mathematics, can be as effective as for all other phenomena observable at our dimensions.

The difficulties intrinsic in an unambiguous interpretation of all aspects of the reality hold also for our language: modern physics, especially in relation to the investigation of the microcosm, warns us that, beyond the domain of our direct observations, our language shows severe limitations. Clear examples of such a situation are concepts like the duality wave-corpuscle, the absence of time and space in extreme conditions of matter, the not empty vacuum, particles going backward in time or not suffering speed limitations or even not existing individually, and so on. Beyond our dimensions, an unlimited region of reality may exists, undisclosed to our 'rational system of thought', although somehow accessible to our 'super-conscious' perceptions.

This should keep cautious those who still defends the idea that everything that cannot be clearly expressed might be wrong or meaningless.

\subsection{Time: the hardest constraint}

Time is actually the most fundamental of the conditions in which we are involved and the one with the most pregnant influence on our common sense and on our language. To hold that there has been a past, that there is a present, and that there will be a future drives all our actions, all our thoughts; all what we think, imagine and realize, all what we speak of, is conditioned by the way we individually experience and represent within ourselves the flow of time.

The way we fit in time - our 'subjective' time - results from the elaboration, by our cerebral processes, of the flow of information coming from the environment: an elaboration tuned with each single organism, which implies important differences among individuals with respect to their modality of perception of time - up to those drastic 'anomalies' consisting in the so called 'altered states of consciousness', typical of pathological or hyper-creative states -, strongly depending on the degree of openness of each person to the environment.

This 'subjective' time - let's denominate it $t_{s}$ - is the main foundation upon which we build our knowledge of nature through our continuous and inescapable interaction with it, and it is also the mediator between us and the world (according to the most recent researches in the neurophysiological field), a possible key factor of the modulation between the components of the cerebral structure, on which our consciousness is grounded [2]. As such, time has to be considered the hardest of all constraints that filter our deep knowledge of the reality.

\subsection{Lack of autonomy of formal systems}

In addition to the difficulties to correctly interpret reality, caused by the influence of both 'soft' and 'hard' constraints, our rationality suffers of a very important shortcoming, characteristic of all formal systems. It consists in the fact that, according to the Kurt Gödel's completeness theorems, autonomous, self-grounded, formal system i.e. capable to not depend on anything external to the system itself - do not exist. For what is here of interest, 
Gödel's achievements stress that the conclusion of any logical process depends, not only on the correct application of its internal rules, but also on propositions and assumptions that escape any internal demonstration.

Therefore, admitted (and not necessarily granted) that the logic-mathematic fabric implicit in our scientific method could be a formal system empty of fails, and considering that the second and the third aspects of our rational system of thought are even weaker than the scientific method (corresponding to the first aspect), on the basis of the Gödel's completeness theorems and taking into account the constant presence of filters of any kind, we are not authorized to always raise to the rank of safe truths all the conclusions derived from our rational elaborations. It is interesting to recall here that, already in the seventeen's century, Blaise Pascal had expressed in his Thoughts the idea that at the top of any logic reasoning there is always a not provable statement from which everything is logically derived.

The limitations of the scientific approach due to the failure of our 'common sense' beyond the domain of our dimensions and to the difficulty to objectify time, together with the lack of autonomy implied by the Gödel's theorems, add to each other, strengthening our suspects that some regions of the reality might be destined to remain at all unveiled by only using our 'rational system of thought'.

In view of these considerations, it is better to be cautious when magnifying the 'comprehensibility of the world' so much referred by some authors, but just remember the David Böhm's explicate and implicate order - the knowable and the unknowable - implying the question whether it is really possible to achieve (even just theoretically) the mythical full objectivity of knowledge, always pursued by science. Let us recall David Bohm when writes that «[...] every kind of thought, mathematics included, is an abstraction, which does not and cannot cover the whole of reality. [...] By giving so much emphasis on mathematics, science seems to be losing sight of the wider context of its vision» [7].

\section{From timelessness to arrows of time}

Let us now have a closer look at the concept of time. With reference to some recent achievements of mathematicalphysics, where dynamical situations in absence of time are hypothesized in the pre-physical phase of the evolution of the Universe (see, for example [3]), it has been suggested that time has emerged to existence, together with space, after the Big Bang (see, for example [8]), then driving its evolution, presently characterized by a cosmological arrow directed toward its expansion. Besides the cosmological arrow, however, physics acknowledges also a thermodynamic arrow, directed from order toward disorder, but neither of the two arrows are contained in the equations of mechanics, where $t$, in all physical theories (classical, relativistic, quantum), is actually schematized as a parameter constituted by an infinite series of unstructured, all equal, instants, linearly following to each other, without any favored direction and where the 'now', the moment in which we can concretely act, is not defined.

The presence of these two arrows does not exhausts the complexity of the concept of time, perhaps the thicker part of the 'Isis's veil', the one under which - according to Heraclitus - Nature loves to hide. Along the evolution of the universe, we observe the continuous birth and death of individual 'islands of complexity', identified in systems in reciprocal interaction with the environment, where the thermodynamic arrow flows for a certain time in the inverse direction (from disorder to order), until when the gradient in direction of order changes sign and disorder eventually wins with the decomposition and the death of the system and the start of a new cycle disorderorder-disorder for an indefinite time. Such 'islands of complexity' - first conceived by Ludwig Boltzmann [9] and then studied in details in the non-equilibrium thermodynamics by Ilya Prigogine - are those in which, by means of our fleeting presence (and of other thinkable living organisms in the vastness of the cosmos) the universe investigates and understands itself.

This difficulty of physics to represent such an important characteristic of time stimulated Ilya Prigogine to consider the necessity to integrate the flow of time within the body of physics [10]. Up to now, however, such an integration has revealed to be a not easy task and the only (although interesting) attempts along this direction refer to the possibility to establish more or less approximated models of the behavior of the subjective time, the only concrete concept of time available to us. For example, the mathematical model on which Metod Saniga is working since many years has been able to geometrically represent an interesting bi-dimensional map of the regions of past, present and future, together with some of the best known anomalies in the perception of time [11], without any reference, however, to the human brain processes and without the possibility to draw from the model new scientific information empirically verifiable/falsifiable. Particularly interesting, for its descriptive details, is the Susie Vrobel's model of 'fractal time' where the present - the 'now' - is structured as a system of several nested 'nows' corresponding to always lower levels of description, each level including retention, consciousness of the present and protentions, fractally organized [12].

Maybe, avoiding any easy identification of the concepts of time underlying $t$ and $t_{s}$ due to the drastic mismatch between them, and considering the fundamental role played by time in the human's knowledge, it is useful to accept the former as the best possible representation of time, not otherwise describable by models derived from the study of the behavior of the subjective time $t_{s}$. Such an approximation is the only possible one and is justified by the important results obtained by using $t$ (despite its alienation from relation) in the descriptions of the fundamental 
laws of nature, unanimously acknowledged by the whole scientific community. We may, then, tentatively connect the two concepts by considering the 'subjective' time $t_{s}$ as a human, individual, modality to represent and handle the 'external', 'objective' (but unknown), time that drives the evolution of the Universe (and then of our existences) [13], variably measurable in different cosmological reference systems.

\section{Two cerebral hemispheres/two logics}

One of the most important peculiarities of our brain is its bipolar, functionally asymmetric, organization in two interconnected hemispheres, interacting to each other through the dense and complex net of nervous paths of the brain's commissure, with the temporization mechanisms playing an essential role:

- the left hemisphere, containing the Broca's area, responsible of the language and, consequently of the linear processes pertaining to the logic-mathematic rationality 'recently' emerged in the human evolution;

- the right hemisphere, containing an area apt to elaborate an amplified somatic consciousness related to the emotions, mostly managing non-linear processes (active since primordial times), implicit in a globalparticipative perception of the environment, impossible to describe with the linear, apophantic, language prevailing to-day.

Actually, the two brain's modules cannot work independently from one another; instead, a continuous harmonic interaction, temporally fine-tuned, is necessary between them for an effective integration of their complementary functions. By means of such a connection, the 'rational' activity - disposed to a timeless and detached quantitative measure able to neglect the subjective, emotional, dimension - may benefit of the intuitional creative inputs coming from the contralateral modality, thus catching the qualitative aspects of the reality, merged in the live flow of time.

It is possible that such a dichotomous feature of the brain could correspond to the system governing our way to store and analyze the information exchanged with the environment; system that, according to Matte Blanco's theory [14], works with two different logics:

- the first logic is mostly connected with more recently exchanged data, stored and elaborated in the same linear, asymmetric form, of our 'rational' system of thought; this kind of information would generally maintain the same logic when emerging out (wittingly or unwittingly) from the unconscious to consciousness;

- the second logic is mostly connected with much older information, deeply hidden in the unconscious, and collected according to the 'generalization principle' and the 'symmetry principle', both strongly characterizing the relation and the 'undifferentiated'. This unforeseen and surprising information coming almost spontaneously from the unconscious cannot generally be elaborated consciously, using the usual tools of our linear logic.

The two logics are irreducible to each other, but a mixture of them may sometimes appears (as it is shown by the structure of some dreams), possibly ascribable to the complex modulation acted by the asymmetric bipolar cerebral structure.

This organization of the brain and the role played in it by time - as the main tool able to modify its quality by effectively combining (in an infinite variety of ways) our rational and relational skills - suggests that we cannot consider time as a normal physical 'observable' to objectify my means of specific experiments, in agreement with the philosophic line of thought of the scientific realism.

\section{5 'Time Machines'}

Newtonian physics displayed a profound difference between the three space dimensions, where any kind of trajectory was possible, and the single time dimension, neatly separated and unmixable with the former, but where forth and back directions were indistinguishable. Moreover, no limit was envisaged for transmission of signals. With the advent of the Relativity Theory the situation changed: in the Einstein's world, time is mixed up with space in four-dimensional manifolds, and a limit to signal transmissions is found, giving rise to the relativity of both time measurement and simultaneity with respect to different observers. Furthermore, when observed from a moving reference system, distances contract and time slows down, with the possibility to imagine time journeys toward future. In spite of these, although very important, changes, nothing has changed, from Newtonian to relativistic mechanics, concerning the reversibility of time $t$, and therefore concerning the possibility to represent mathematically the past and the future.

In one of the possible versions of the twin 'paradox', Amy, at the age of 21, leaves her twin brother, Max on the Earth and start for a two-ways trip toward GJ832 - a red dwarf star distant 16 light years from Earth - with a spaceship, at a speed of about $2300000 \mathrm{~km} / \mathrm{s}(\sim 77 \%$ of the velocity of light), in order to have a close look at GJ832b, a Jupiter-like planet of that planetary system [15].

Taking into account that the reference system related to Earth is an inertial one, while it is not so for the reference system related to the spaceship, the calculations - independently done by each of the twins - show that, when Amy returns to Earth after 42 years, she finds herself to be only 48 years old and therefore 15 years younger than Max, already at the age of 63. This is what the calculations say, on the bases of the dilation observed by each of them on the other's time, on the contraction of the distance between Earth-GJ832 as observed from the moving 
spaceship, and on the limited speed of the signals that the two brothers send to each other in order to be kept continuously informed [16].

Upon arrival on the Earth, if the fifteen years difference between her and Max (and with all other humans in the Earth) would be biologically visible in their bodies, Amy, being aware of it, would agree to have landed in the future of Max, independently of the appearance of the country, completely changed after the start of the trip, 42 (solar) years ago. In this case - the same as being hibernated for 15 years - Amy's feeling to be in the future of her place would not have only a psychological sense since her biologic processes would have really stopped for that time.

In the opposite case, with no visible biological changes appearing to Amy, with respect to Max, upon her arrival on the Earth, her only consideration can be to observe the changes occurred in the country (changes that have, instead, being continuously monitored by Max), and her feeling to be in the future of Max would only have a psychological sense. This kind of feeling, in fact, may not necessarily come from a trip at relativistic speed; it may simply derive from the very fact of having remained far away from her country for long time, as is normally the case.

Concretely, there is no way to calculate or to know whether or not any biological changes, to be visible upon arrival on the Earth, occurred in the traveller's body along the trip, and therefore, by considering that the time $t$ used by the Relativity Theory for proposing Time Machines is not the time $t_{s}$ by means of which humans evaluate their time evolution, no empirical check is presently possible on the reliability of the application of this theory to human travels. Even stronger concerns apply to the theory of human travels to the past. In the context of the General Theory of Relativity, it is hypothesized the existence of a 'time inversion region' in the vicinity of a nude singularity, where time may invert its flow with respect to that measured at a great distance. Such a condition makes theoretically possible that a traveler $A$ leaving the Earth and returning back after having crossed this 'time inversion region', may experiment a drastically different temporal evolution, showing a large difference of age with respect to an observer $B$ left on the Earth; difference of age that, according to de Felice, corresponds to $A$ traveling in the past or in the future of $B[17$, p. 203]. Basing on the discovery of the existence of closed light-type geodesics including potential walls within the 'time inversion region' [18], the same Author derives that light photons may enter this region, stay there as long as requested to recover all the necessary time, and then, pushed by the potential wall, may return back to Earth at the same time in which they left [17, p. 209]. This achievement shows also, according to de Felice, that it is not possible that a probe, sent toward a 'time inversion region', could return back to the past of the Earth [17, p. 203 and p. 209].

The involved matter is quite complex - both from a theoretical and experimental point of views - and extreme care is required for precise evaluations. For the time being, and waiting for new investigations aimed to resolve the still existing doubts (especially those concerning the proposed solutions of the paradoxes implied by the travels in the past), we want here to keep aside the unrealistic possibility to use 'wormholes' of the spacetime in order to jump to the past or the future of a place, for the not negligible fact that the human physical structure is unable to sustain the enormous forces existing in the vicinity of a singularity. On the other hand, however, an inverted temporal sequence (change of simultaneity) of a phenomenon observed by two different observers - both in the cases foreseen by the Special Relativity and in those derived by the General Relativity concerning the presence of 'time inversion regions' [17, pp. 211-213] - does not mean tout court that one of the observers is in the past or in the future of the other, and, especially, that the one can act in the past of the other. In fact, one thing is to observe a time inversion between two phenomena by looking at the arrival of signals from a distant place (even from a 'time inversion region'), another, much different thing, is to talk of a human being who goes to the 'time inversion region' and comes back older, because in this case, as already stressed above, we have to take seriously into account the human's biology and his 'subjective' time.

Actually, the above considerations seems to suggests that a human being is subject, during a high speed interstellar trip, to the need to successively modify his representation of the 'external' time, when jumping in various reference systems - from the Earth to the spaceship and vice versa, with changing speed, and approaching to or leaving an intense gravitational field - and to the corresponding need to manage these modifications, safely for his body. Therefore, if our 'subjective' time $t_{s}$ is connected to the 'real' time which drives the evolution of the universe (through our body's senses and through our brain that use the former in conformity of its structure and demands), we cannot avoid to consider the foreseen biological changes (in particular, concerning $t_{s}$ ) to which the human-observer is subject during an interstellar trip.

We conclude that, given the present impossibility to evaluate the necessary but unknown changes of the 'subjective' time - the one that connects us to the reality, the fundamental one on which we have to rely for anything related to our interpretation of external phenomena - along the two-ways trip far away from the Earth, the Time Machines hypothesized in the context of the Theory of Relativity are nowadays only a fascinating theoretical possibility, certainly good for art and fiction, but not of concrete realization. We believe that the discrepancy between the theoretical expectations derived from the Theory of Relativity and the practical realization of a Time Machine - i.e. machines able to bring us to our past or to our future and to operate there as if we were in our present - depends on the fact that the theory uses a time $t$ which has nothing to do with the lived, 'subjective', time experienced by humans, the ones who pretend to overcome the direction of flow of time, normally going from past to future. The theoretical representation of time by physics by means of the time-parameter $t$, at all appropriate for 
describing the fundamental laws of physics, appears inadequate for use in the place of the subjective time $t_{s}$ when theorizing Time Machines available to human beings for transportation in their past or in their future. The questionability is immediately realized if we look at time from the point of view of the subjective experience where the human aspects related to relation and participation are taken into account.

\section{From 'endophysics' to a 'complex thought'}

In the eighties of last century, with the discovery of the interacting role of the observer by Quantum Mechanics, it was felt the need of an Endophysics - a physics with the world seen from within and able to include interaction, disregarded by the scientific realism - as opposed to the standard non-relational classical physics, denominated Exophysics [19]. In this new perspective, the study of nature should have systematically included man as part of it, being he a self-organized system, produced by nature but strictly interacting with his environment from which he receives the necessary physical and informational feeding.

At that time, the construction of an Endophysics appeared as the picklock for the solution of the problem, with the possibility to formulate new physical theories with full meaning from the human standpoint and able to contribute to the solution of the paradoxes proposed by MQ. Unfortunately, such a construction has revealed to be an impossible enterprise: the available advanced mathematical techniques, born and developed within the exophysical paradigm of which they are integral part, are not appropriate to pursue such an objective. Our mathematics, divorced from relation, even able to express the man's rational skills by well modeling the external reality, cannot include him explicitly, thus hampering the construction of an operative Endophysics [20]. This is probably one of the most important reasons why the passionate debate about Endophysics switched off after a very intense explosion of research activities, forcing, de facto, Exophysics as the only possible investigational approach.

Taking into account the failure of the debate about Endophysics, we should try other ways, aiming to gain a new perspective, in order to reconcile the need to not neglect interaction with the difficulty to build a new mathematics able to include explicitly the observer in his nature's investigation.

At this purpose, taking into account the already achieved awareness that a real conceptual jump beyond our unavoidable cultural and psychological schemas is only possible by seriously challenging our rigid convictions, in order to gain, even at a price of a hard and painful confrontation towards ourselves, an ampler and more comprehensive view, we think appropriate to look deeper at the concept of rationality - also with reference to the current trends, and benefitting of the extended discussions had on this topic in three International Meetings held in Palermo (1999), Tatranská Lomnica (2002) and Bielefeld (2005) [21] - with the aim to revitalize the debate on the need of a change of paradigm.

Our proposal intends to go beyond the endophysical perspective by skipping the search for a new, improbable, mathematical system where the experimenter is included in its formulas, but where it is accepted the anthropologic datum that at the downing of the civilization the acquisition of knowledge was based on the process of cooscillatory resonance that the human body established with his environment, as an effect of a hearing amplified by the whole organism and transmitted to the emerging consciousness of the future observer. By taking into account these peculiarities of the human body and of his brain, it becomes overriding the need to understand how to achieve a responsible recovery of the perceptual characteristics of empathic-imitative type that have been constantly reduced with the secular exercise of the rationality, in order to foster a new equilibrium between the complementary skills of the human being. One of the most relevant consequences of such a recovery would be a far-reaching change in our idea of time, since man, effectively using the contralateral skills, would be able to integrate the temporal dimension in terms of a participatory adhesion to the present, nowadays excluded from science's processes of knowledge.

Therefore, without neglecting the need to search for always safer conceptual tools in the context of the scientific investigation, we are left with the task to favor the identification of those neglected skills connected with the history of the mankind and with its vital values. These skills, already present in the collective unconscious, are the only ones - within the human possibilities - able to address the correct strategies of our behavior that, once put into operation by means of the mindful rationality, may rule men's actions within an ethical framework of general comfort, built upon the values of participation and reciprocal help. The corresponding gnoseological approach may be nourished by the human conflicting restlessness able to welcome and effectively use the opposition between the antithetic possibilities of the being.

We should to 'reset' again our system (to use a computer science terminology), going to recover the synthetic aspect of the pre-logic modality of knowledge with its high artistic-symbolic content, able to generate enough tensive energy to feed - through the conflict between the opposite poles - the trigger of those high tension sparks on which the human creativity has always based itself: a sort of 'Court of Gentiles' within the privacy of our own consciousness, as a space of unconditioned hospitality of everybody else, even included the mythical dimension of those populations not yet resigned to our self-defined 'civilizing' action, as well as of the man's infancy needing a fairy-like dimension, both free from the coaction to any 'measurement', characterizing the scientific rationality.

In view of the need to look for a new gnoseologic paradigm and of the mentioned limitations of our 'rationality', with particular reference to the difficulty to 'objectify' the flowing of time from past to future, we are 
encouraged to acknowledge those contralateral cognitive possibilities, nowadays discarded but to which man has always counted (often unwittingly): think, for example, to the masterpieces of great artists, scientists and mystics of any epoch and culture, able to empathically disclose the underlying principles of life by deep and sincere introspection, and to communicate them with touching expressiveness. We then consider necessary, and have accordingly proposed, a model for a new rationality - justified by the achievements of the modern physics -, ampler with respect to the totalizing one, prevailing to-day; a 'complex' thought that - pulling back into play the contralateral thought (the effusive-participative modality in addition to empiricism and logic-mathematics) - could contribute to temper the exuberances of the scientific rationality, favoring its revitalization [22].

It is for us thinkable that the harmonic confluence of the two modalities of thought - the logic one with its capability of abstraction from the reality, and the participative one with its capability of deep introspection, separately pursued by science and art respectively - could significantly power our thought, allowing a knowledge closer to an 'absolute' one, only subject to the awareness of our physical and psychical limitations (particularly our limited language and common sense) for which such knowledge may be conveyable only partially and never in its entirety and objectiveness, in the sense attributed to this term by science. Indeed, being aware of such limitations, and wittingly giving up about the search of an absolute objectivity, never reachable anyway, all regions of the reality (even beyond our physical dimensions) maybe investigated and analyzed in this way.

It is important to underline that such a 'complex thought' is grounded on the harmonization of the human system in the integrality of its constituent elements, even if they are opposite and irreducible to each other. The reason lies in the consideration that $\alpha \rho \mu$ oví $\alpha$ is mainly $\pi$ ó $\lambda \varepsilon \mu \rho \varsigma$ and not the false peace of the sloth and of the disownment of differences, since any difference will inevitably emerge anytime a dialogue is established between individuals in painful and tensive contraposition: a sort of faustum proelium, converging in a coherent recomposition of consonances and concordances in a region conterminal to both the poles of the relation and therefore unpredictable from each single perspective. A faustum proelium, then, able to produce a highly evolved gnoseological model of knowledge (occasionally manifested only by most ingenious and innovative personalities), and to positively comply with the mysterious cleavage through which the scholars welcome, without being charmed, the voices of the Muses, symbolized by the song of the cicadas who accompany the Fedro's extraordinary dialogue with their high, undetermined, frequencies. At this purpose, it is worth noticing that Plato tells us that the dialectic orality, prerequisite of the philosophic logos, has a dynamis propulsive and incremental of knowledge, while the mimetic-poetic orality - based on myths and images - characterizes poets and rhapsodes active (better, passive!) for obsession and divine inspiration, not for science. This concept is also stressed by Socrates when says that: «Until when he has full control of his faculties, no one is able to write poetries or vaticinate» [23].

On one side, the Orpheus' imitative-participatory skills assert themselves, at the expenses of his own consciousness, on the level of the keenest expressive shadows of the reality, while, on the other side, in the promethean impetus of science, the identity principle, foundation of a logos capable of quantitative evaluations allowing him to dominate the world! - is established by means of his shrinking from the participation, with the aim to reach to a most possible objective and linear knowledge.

It is intriguing to look at the harmonization aspect from the perspective of a musical mind - as expression of the development of the human consciousness - by considering that the same construction and practice of the musical instruments has always presupposed the use of both cerebral hemispheres. Music - actually the art/science of the organization of sounds in the temporal dimension - is produced by a hybridization-collaboration of the two complementary mental activities, i.e. the ancestral emphatic-imitative knowledge with the rational and analytical skills. On the basis of our proposal, it can be easily understood why the musician, in front of the option whether to be Prometheus or Orpheus, should not necessarily lean toward the prototypic figure represented by the Thracian poet but, on the other side, it can also be shown that his innate restlessness (of which music is both expression and therapy) is neither recognizable in the antipodal and detached Prometheus. In fact, since music is nourished with time while living in the present, the temporal diffraction represented by the system Prometheus /Epimetheus ('the one who see before' and 'the one who sees after'), is just missing the present, otherwise necessary for Elpis, the Hope, for her impetus toward future. Hope, in this way, would be blocked in her motion and imprisoned in the bottom of the jar, as myth has invariably recorded.

Our system, as it is, would tend to push us to an oscillation between a 'Promethean' control of nature, able to fit our aims, and the opposite availability to an (even transient) 'Orphic' appropriation that cannot be carrier of true knowledge for its tendency to dispossess the subject of his self-control. Glaring evidence of our claim is the historic-anthropologic condition of our society, both highly technologic and always more committed to the dispossessing of its own rationality by the use of the various psychotropic stuffs.

So, since both are missing something - Orpheus the 'rationality', and Prometheus the 'relationality' -, then, for the amphibious and mediating character of music (that is, of a well-tempered cerebral activity), specialized in the transit of information between the opposite poles of human mind, we all should opt for the reception in toto of the full system, susceptible of harmonization. The 'musician', in fact, being conditioned' by the same instrumental practice to a simultaneous use of both hemispheres, does not underestimate any of the two poles, nor he lives them alternately; on the contrary, he tries to welcome - at the same time - both the $\lambda$ ó $\gamma$ os, given by the stability of the first pole, and the $\pi \alpha \dot{\alpha} \theta \mathrm{o} \varsigma$, given by the oscillation of the second one. Instead of pendulating between the extremes, 
he connects them instantaneously, therefore taking permanently part of both, as it happens in the stable trembling of the live flame of a candle.

By considering that the neural correlates of the consciousness show a dual asymmetric feature, it appears justified to infer that the world's knowledge, by means of them, would greatly benefit from the man's adaptation to such a complexity with a suitable optimization of the whole system.

Now, assuming that each human being may be free to address his own consciousness to all the possibilities included between the two extremes of a 'blind' relationality lacking of any rationality and a 'deaf' rationality detached from the environment, it is not unreasonable to maintain that the choice of either of the two opposite poles is the most direct way to exercise a violence toward the environments, determining a situation of non-equilibrium, obviously portending, in a long term, to a collective destruction. Therefore, if it is true that any approach to knowledge may be judged on the bases of its results (i.e. if it is able or not to reach the mankind's telos), and if we assume that the more obvious telos is the survival of the mankind, we cannot lean toward any of the two extreme poles, but insist for finding a bridge between them, able to exploit in the most effective way their complementarity.

It is worth to underline, at this purpose, that time is indeed the key factor of such a fine regulation process of the gnoseologic human potential. Therefore, by choosing to enhance one of the two aspects with respect to the contralateral one, ends with excluding the 'pure living in the now', tuned with the flow of time, or the 'pure thinking' in that detaching state, capable to guarantee the awareness of the before and the after. In these conditions, to think to a physical Time Machine means to support a paradox, because a 'machine' plays normally the role of a device integrated with the human body and apt to 'prolong' an organ of his, whereas, in this case, the 'machine', built using a time $t$ lacking integration (and then detached from external reality), would only power a 'time out of joint' and not the same potentially integrated human consciousness, the only concrete basis for a real Time Machine.

We suggest, in summary, to overcome both extreme tendencies by postulating a third form of knowledge that, through a 'mediation' between the 'not-rational relationality' of myth and the 'not-relational rationality' of science, would be able to assert the possibility of a 'relational rationality', where our reason may develop in a close alliance with the request for an empathic participation in a sort of concordia discors between the primitive mentality and the scientific rationality.

\section{Conclusions}

The Theory of Relativity has given rise to fantastic ideas about the possible existence of Time Machines with which we could travel to past or to future and to act there. However, their theoretical realizations are not based on the 'subjective' time $t_{s}$ experienced by man, as it should, but on the simple linear parameter, $t$, of the mathematical equations of the relativistic mechanics, representing a time (like in the Newtonian mechanics) without any internal structure or a favorite direction, presupposing absence of interaction, and assumed to be representative of the 'real' ontological 'objective' time that drives the evolution of the universe.

Indeed, the parameter $t$ is just a theoretical tool, very effective in many respects, but not in the case in which it is used in substitution of a full human experience, if we consider that this tool fully disagrees with the human, interactive, subjective time, equipped with a very complex structure, variable from an individual to the next, and able, within the internal consciousness of each of us, to slow down, to accelerate or even to invert its direction, as it happens in our dreams or in several 'altered states of consciousness', where the linear logic of the scientific rationality does not hold.

A Time Machine based on $t$ is intrinsically grounded on the same presumption of the scientific rationality that prevents to glimpse the nucleus of the solution by ignoring the fact that any interaction can only occur in time and by means of time. We are products of nature on the Earth and absorb times and rhythms produced by nature on the Earth, or observed on the Earth even if produced outside. Our physical system is based on such rhythms, particularly our brain, subdivided into two cerebral hemispheres in strict connection to each other, where time and these rhythms play a paramount role for our acquisition of knowledge. Susie Vrobel writes: «The fractal geodesics of spacetime as an ontological entity act as a constraint on our microscopic physical makeup and therefore on our experience. Embodied brains subjected to this and other constraints design experiments and develop models and theories of spacetime. It is our simultaneous participation to both the quantum and the classical level which forms the prerequisite for insight and cognition. Phenomenology and ontology seem to become inextricably intertwined» [24].

To use $t$ instead of $t_{s}$ cannot give account of that real Time Machine, based on our individual consciousness, which is capable to prepare oneself (although unwittingly) to a simultaneous gathering of information coming from all over the cosmos - present, past and future - together with the ability to make previsions, and to allow anyone of us to make observations and measurements in our present, while taking care of the past and the future. Having excluded both interaction and subjective consciousness and, at the same time, pretending to have understood of the nature of time - the same time in which man is put into being and comprehends the universe that has produced him - creates the paradox to believe to have allowed man to be able to travel in that same dimension in which the entire epistemologic project of science is against; i.e. his introduction and his consequent movement in a not-human time. 
Pretending paradoxically to move man from a point to another of the river of time, while maintaining him dry from the water of consciousness and interaction, appear to us to be an interesting literary or artistic expedient, but not the perspective of a real human experience.

A 'real' ( $t_{s}$-based) Time Machine could only arise by integrating $t_{s}$ in the body of a new kind of rationality - a 'complex thought' - where empiricism and logic-mathematic are harmonized with participation and interaction [25]. The goal might be achieved - in our opinion - if instead of pursuing the objectivity for a presumed universal knowledge, science should base itself on an universalization of man in the knowledge process. For this purpose, man has to use his whole mind, in all its components, harmonized by means of time. As we derive from Plato's Timeo, the true music does not lie in the production of organized sound, vocal or instrumental, but on the condition of the subject in his relationship with the world. All the same, if the aim of man is the knowledge of the laws governing the universe (that are internally contained in himself), it is up to him to recognize them within himself, thus putting back 'time in joint'.

The efforts of the ongoing joint research in neurophysiology and physics - without neglecting any important contribution coming from anthropology - could perhaps give new answers in the future.

\section{References}

1. W.J. Ong, Orality and Literacy: The Technologizing of the Word (London [u.a.], Methuen, 1982)

2. A.R. Damasio, Emozione e coscienza (Milano, Adelphi, 2000, 239-240; orig. ed., The Feeling of What Happens: Body and Emotion in the Making of Consciousness (New York [u.a.], Harcourt Brace, 1999)

3. M. Heller, Tensione Creativa: Saggi sulla scienza e sulla religione, Ferrara, AKOUSMATA · orizzonti dell'ascolto, 2012, 287-290; orig. ed., Creative Tension: Essays on Science and Religion, PhiladelphiaLondon, Templeton Foundation Press , 2003)

4. M. Heidegger, Nietzsche (Milano, Adelphi 2000, 642-670; orig. ed. Nietzsche, I-II, Pfullingen, Neske , 1961)

5. H.-G. Gadamer, Verità e metodo (Milano, Bompiani, 1983, 342-357; orig. ed., Wahrheit und Methode: Grundzüge einer philosophischen Hermeneutik, Tübingen, Mohr , 1960)

6. G. Schröder, The Hidden Face of God: How Science Reveals the Ultimate Truth (Schröder, New York [u.a.], Free Press, 2001)

7. D. Bohm, F.D. Peat, Science, Order, and Creativity (London-New York, Routledge, 2005, 9-10; first ed. Toronto [u.a.], Bantam, 1987)

8. F. de Felice, Cosmogon: Alla ricerca delle ragioni dell'essere (Ferrara, AKOUSMATA • orizzonti dell'ascolto, 2012)

9. L. Boltzmann, Nature, 51, 1322 (1895), pp. 413-415

10. I. Prigogine, La fine delle certezze: Il tempo, il caos e le leggi della natura (Torino, Bollati Boringhieri, 1996)

11. M. Saniga, Algebraic Geometry: A Tool for Resolving the Enigma of Time?, in R. Buccheri, V. Di Gesù and M. Saniga (eds.), Studies on the Structure of Time: From Physics to Psycho(patho)logy, (New York [u.a.], Kluwer Academic/Plenum Publishers, 2000), 137-166

12.S. Vrobel, Fractal Time. Why a Watched Kettle Never Boils (New Jersey, World Scientific , 2011), 13-33

13. M. Alfano, R. Buccheri, Temporalità e interazione come strumenti di analisi in una prospettiva endofisica della conoscenza, in M. Alfano e R. Buccheri (eds.), Tempo della Fisica e Tempo dell'Uomo: Relatività e Relazionalità, Ferrara, AKOUSMATA · orizzonti dell'ascolto, 100-101 (2009),

14. I. Matte Blanco, The Unconscious as Infinite Sets: An Essay in Bi-logic (London, Duckworth, 1975)

15. J. Bailey, et alii, Astrophys.J. 690, 743-747 (2009)

16. F. de Felice, L'intreccio spazio-temporale: La relatività dello spazio e del tempo: la sua origine e il suo mistero (Torino, Bollati Boringhieri, 2006), 130-135

17.F. de Felice, Gli incerti confini del cosmo: dai buchi neri alle macchine del tempo (Milano, Mondadori, 2000)

18.F. de Felice, M. Calvani, Gen. Rel. Grav., 10, 4, 335-342 (1979)

19. O.E. Rössler, Endophysics: The World as an Interface, Singapore [u.a.], World Scientific (1998), p. 27

20. R. Buccheri, G. Jaroszkiewicz, M. Saniga, Recent Res. Devel. Astronomy \& Astrophys, 1 , 609-623: 621 (2003)

21.R. Buccheri, V. Di Gesù, M. Saniga (eds.), Studies on the Structure of Time: From Physics to Psycho(patho)logy, New York [u.a.], Kluwer Academic/Plenum Publishers (2000); R. Buccheri, M. Saniga, W. M. Stuckey (eds.), The Nature of Time: Geometry, Physics and Perception, Dordrecht [u.a.], 
Kluwer Academic (2003); R. Buccheri, A.C. Elitzur, M. Saniga (eds.), Endophysics, Time, Quantum and the Subjective, Singapore [u.a.], World Scientific (2005)

22. M. Alfano, R. Buccheri, Lateranum, 78, 2, 245-266, 2012

23. G. Reale, Introduzione (Platone, Fedro, Milano, Valla-Mondadori, 2005), XV

24.S. Vrobel, Fractal Time and Fractal Spacetime: Phenomenology or Ontology?, in Ji-Huan He (ed.), Fractal Spacetime and Noncommutative Geometry in Quantum and High Energy Physics, I/1, Hongkong, Asian Academic Publisher Limited (2011), 55-58

25. M. Alfano, R. Buccheri, Toward a 'rationality' tuned with human life (Vilnius, July 2012 , to be published) 
